
\title{
25 Research Soure \\ Electric field control of spin-orbit torque switching in a spin-orbit ferromagnet single layer
}

\section{Miao Jiang}

Department of Electrical Engineering and Information Systems, The University of Tokyo

\section{Hirokatsu Asahara}

Department of Electrical Engineering and Information Systems, The University of Tokyo

\section{Shinobu Ohya}

The University of Tokyo https://orcid.org/0000-0003-1417-9403

\section{Masaaki Tanaka ( $\nabla$ masaaki@ee.t.u-tokyo.ac.jp )}

The University of Tokyo https://orcid.org/0000-0003-3599-663X

\section{Article}

Keywords: Multifunctional Spin Logic, Memory Devices, Spin-orbit Ferromagnet, Carrier Concentration Modulation

Posted Date: December 14th, 2020

DOl: https://doi.org/10.21203/rs.3.rs-106873/v1

License: (c) (1) This work is licensed under a Creative Commons Attribution 4.0 International License. Read Full License 

10

Electric field control of spin-orbit torque switching in a spin-orbit ferromagnet single layer Miao Jiang $^{1 *}$, Hirokatsu Asahara ${ }^{1}$, Shinobu Ohya ${ }^{1,2,3^{*}}$ and Masaaki Tanaka $a^{1,2^{*}}$

${ }^{1}$ Department of Electrical Engineering and Information Systems, The University of Tokyo, 7-3-1 Hongo, Bunkyo-ku, Tokyo 113-8656, Japan

${ }^{2}$ Center for Spintronics Research Network (CSRN), Graduate School of Engineering, The University of Tokyo, 7-3-1 Hongo, Bunkyo-ku, Tokyo 113-8656, Japan

${ }^{3}$ Institute of Engineering Innovation, Graduate School of Engineering, The University of Tokyo, 7-3-1 Hongo, Bunkyo-ku, Tokyo 113-8656, Japan

*E-mail: jiangmiao@g.ecc.u-tokyo.ac.jp; ohya@cryst.t.u-tokyo.ac.jp; masaaki@ee.t.u-tokyo.ac.jp

To achieve a desirable magnitude of spin-orbit torque (SOT) switching and realise multifunctional spin logic and memory devices utilising SOT, controlling the SOT manipulation is vitally important. In conventional SOT bilayer systems, researchers have tried to control the switching behaviour via interfacial oxidisation; however, the switching efficiency is limited by the interface quality. A current-induced effective magnetic field in a single layer of a ferromagnet with strong spin-orbit interactions, the so-called spin-orbit ferromagnet, can be utilised to induce SOT. In spin-orbit ferromagnet systems, electric field application has potential for manipulating the spin-orbit interactions via carrier concentration modulation. In this work, we demonstrate that SOT switching can be successfully controlled via an external electric field using a (Ga,Mn)As single layer. By applying a gate voltage, the switching current density can be solidly and reversibly manipulated with a large ratio of $14.5 \%$, which is ascribed to the successful modulation of the interfacial electric field. Our findings help further the understanding of the magnetisation switching mechanism and advance the development of gate-controlled SOT devices. 
Spin-orbit torque (SOT) has been proposed as a promising candidate to control the

2 magnetisation of ferromagnetic materials, showing great potential for realisation of spintronic memory devices with better stability, higher scalability, faster information processing speed and lower energy consumption. To further enhance the SOT magnetisation switching efficiency, researchers have explored various effective methods. First, the most generally used approach is to find materials with a large spin Hall angle $\theta_{\text {sH }}$ that can generate a large spin current, which can be utilised to effectively decrease the critical switching current density $J_{\mathrm{c}}{ }^{1,2}$. Those materials with large $\theta_{\mathrm{SH}}$ are utilised in bilayer systems, where the spin current generated in a nonmagnetic layer is injected into the adjacent ferromagnetic layer and exerts a torque to switch the magnetisation. In bilayer systems, the SOT switching can be manipulated by controlling the interfacial oxidisation $^{3-6}$. However, the spin injection is limited by the interface quality, which hinders further decreases in $J_{\text {c. }}$ To overcome this limitation, a new class of materials called spin-orbit ferromagnets, including ferromagnetic topological insulators and the ferromagnetic semiconductor Mn-doped GaAs ((Ga,Mn)As), have recently been found to be very promising for highly efficient magnetisation switching due to the strong spin-orbit-induced effective magnetic field and the large spin polarisation ${ }^{7-10}$. Especially in $(\mathrm{Ga}, \mathrm{Mn}) \mathrm{As}$, an extremely low $J_{\mathrm{c}}\left(4.6 \times 10^{4}\right.$ $\mathrm{A} \mathrm{cm}^{-2}$ ) has been demonstrated by suppressing the contribution of the field-like term with a current-induced magnetic field ${ }^{11}$. In ferromagnetic topological insulators, electric field application has been demonstrated to control $J_{\mathrm{c}}$ via modulation of the density and type of surface carriers $^{10,12-13}$. However, the switching process is still improvable in terms of the switching hysteresis and its completeness ${ }^{10}$. To achieve a desirable magnitude and realise multifunctional SOT spin logic and memory devices with a simple structure and high efficiency, manipulating the SOT in an appropriate spin-orbit ferromagnet single layer is strongly required. The single- 
crystalline ferromagnetic semiconductor $(\mathrm{Ga}, \mathrm{Mn}) \mathrm{As}$ is one of the best material systems for this purpose, where the Dresselhaus and Rashba effective fields ( $H_{\mathrm{D}}$ and $\left.H_{\mathrm{R}}\right)$ induced by the spinorbit interactions contribute to an in-plane spin component that can exert a strong torque on the magnetisation ${ }^{8}$. Because both $H_{\mathrm{D}}$ and $H_{\mathrm{R}}$ are strongly influenced by the internal electric field ${ }^{14,15}$, external electric field application is expected to achieve effective manipulation of the SOT in (Ga,Mn)As.

In this Article, we demonstrate that the SOT switching behaviour in a ferromagnetic (Ga,Mn)As single layer can be manipulated by applying an electric field using a solid gate electrode, as shown in Fig. 1a. By changing the sign and magnitude of the gate voltage $V_{\mathrm{g}}$, the interfacial electric field and hole concentration of $(\mathrm{Ga}, \mathrm{Mn}) \mathrm{As}$ are manipulated, enhancing or suppressing the spin-orbit interactions. Because the spin-orbit interactions couple the spin of a hole with its momentum and generate $H_{\mathrm{D}}$ and $H_{\mathrm{R}}(\text { Fig. } 1 \mathrm{~b})^{16-19}, H_{\mathrm{D}}$ and $H_{\mathrm{R}}$ are very sensitive to the applied electric field. By applying a positive $V_{\mathrm{g}}$, we show that the enhancement of $H_{\mathrm{D}}$ and $H_{\mathrm{R}}$ contributes to an increase in the SOT switching efficiency, decreasing $J_{\text {c. }}$ Our findings provide a promising method for efficient modulation of SOT switching by applying an electric field to a single spin-orbit ferromagnetic layer.

\section{Magnetisation reversal in the $\mathrm{Au} / \mathrm{Cr} / \mathrm{AlO}_{x} /(\mathrm{Ga}, \mathrm{Mn}) \mathrm{As}$ system}

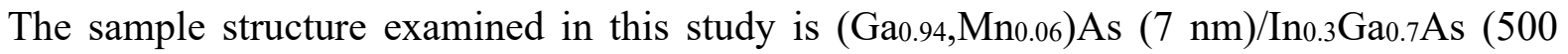
$\mathrm{nm}) / \mathrm{GaAs}(50 \mathrm{~nm})$ grown on a GaAs (001) substrate by molecular beam epitaxy (MBE). Due to the 500-nm-thick lattice-relaxed insulating $\operatorname{In}_{0.3} \mathrm{Gaa}_{0.7} \mathrm{As}$ layer, a tensile strain is applied to the (Ga0.94,Mno.06)As thin film, inducing perpendicular magnetic anisotropy (PMA). As shown in Fig. 1a, the film was patterned into a crossbar (red parts in Fig. 1a) with a size of $0.5 \mu \mathrm{m}$ (width) $\times 2$ 
$1 \mu \mathrm{m}$ (length) by electron beam lithography for transport measurements. The $\mathrm{Au}(100 \mathrm{~nm}) / \mathrm{Cr}(5$

$2 \mathrm{~nm}$ ) electrodes (yellow parts in Fig. 1a), which also work as heat sinks, were deposited with

3 electron beam evaporation. For the solid gate electrode, 40-nm-thick $\mathrm{AlO}_{x}$ was deposited by

4 atomic layer deposition at $150{ }^{\circ} \mathrm{C}$ as a dielectric layer. (See Supplementary Note 1 in the

5 Supplementary Information for the detailed device fabrication process.) Through characterisation

6 of the anomalous Hall effect (AHE), the PMA is confirmed by measuring the Hall resistance $R_{\mathrm{H}}$

7 with sweeping of a magnetic field $H_{z}$ applied perpendicular to the film plane. In Fig. 2a, the $R_{\mathrm{H}}-$

$8 H_{z}$ curve (see the black curve) shows an obvious square-like character, where $R_{\mathrm{H}}$ varies between

9 approximately $\pm 0.6 \mathrm{k} \Omega$. The coercivity $H_{\mathrm{c}}$ is $300 \mathrm{Oe}$, as shown in the square-like $R_{\mathrm{H}}-H_{z}$ curve,

10 and we see an obvious anisotropic magnetoresistance (AMR) effect (see the red curve in Fig. 2a),

11 where $R$ reaches the maximum value when the magnetisation starts to be reversed by $H_{z}$.

12 In addition to a magnetic field, a current $J$ can also be utilised to switch the magnetisation.

13 As shown in Fig. 2b, with the help of a small external magnetic field $H_{\text {ext }}=300$ Oe for

14 deterministic magnetisation reversal, $R_{\mathrm{H}}$ can be changed between $\pm 0.6 \mathrm{k} \Omega$ by sweeping $J$ along

15 the in-plane direction, which is consistent with the AHE result in Fig. 2a; this result indicates that

16 the magnetisation can be fully reversed between the $+z$ and $-z$ directions with a rotation angle of

17180 deg by $J$. For $J / /[\overline{1} 10]$ (see the black curve in Fig. 2b and the illustration of torques in Fig.

$182 \mathrm{c}$ ), the spin component $\widehat{\sigma}_{x}$ induced by the effective field is along the [ $\left.\overline{1} \overline{1} 0\right]$ direction, as shown

19 in Fig. 1b, which exerts an anti-damping torque $\hat{\boldsymbol{\tau}}_{\mathrm{ST}}$ on the magnetic moment. $\hat{\boldsymbol{\tau}}_{\mathrm{ST}}$ is proportional

20 to $\widehat{\mathbf{m}} \times \widehat{\boldsymbol{\sigma}}_{x} \times \widehat{\mathbf{m}}$, and the direction of $\widehat{\boldsymbol{\tau}}_{\mathrm{ST}}$ is the same as that of $\widehat{\boldsymbol{\sigma}}_{x}$. Here, $\widehat{\mathbf{m}}$ represents the unit

21 magnetisation vector. With the assistance of the torque $\hat{\boldsymbol{\tau}}_{\mathrm{ext}}$ induced by $H_{\mathrm{ext}}$, i.e., $\hat{\boldsymbol{\tau}}_{\mathrm{ext}}=-\widehat{\mathbf{m}} \times \widehat{\mathbf{H}}_{\mathrm{ext}}$

22 (here, $\widehat{\mathbf{H}}_{\mathrm{ext}}$ represents the vector of $H_{\mathrm{ext}}$ ), $\hat{\boldsymbol{\tau}}_{\mathrm{ST}}$ overcomes the torque $\hat{\boldsymbol{\tau}}_{\mathrm{an}}$ induced by the

23 perpendicular anisotropy field $H_{\mathrm{an}}$, i.e., $\widehat{\boldsymbol{\tau}}_{\mathrm{an}}=-\widehat{\mathbf{m}} \times \widehat{\mathbf{H}}_{\mathrm{an}}\left(\right.$ here, $\widehat{\mathbf{H}}_{\mathrm{an}}$ represents the vector of $\left.H_{\mathrm{an}}\right)$, 
1 and reverses the magnetic moment when $J$ increases. In the case of $J / /$ [110] (see the orange

2 curve in Fig. 2b and the illustration of torques in Fig. 2d), the induced spin component $\widehat{\sigma}_{y}$ points

3 in the [110] direction, which is parallel to $\hat{\boldsymbol{\tau}}_{\mathrm{ext}}$ and opposite to $\hat{\boldsymbol{\tau}}_{\mathrm{an}}$. Therefore, with increasing $J$,

$4 \hat{\boldsymbol{\tau}}_{\mathrm{ST}}$ is enhanced, and magnetisation reversal occurs. Here, we note that a small hysteresis window

5 appears when $J$ is between $12 \times 10^{5} \mathrm{~A} \mathrm{~cm}^{-2}$ and $17 \times 10^{5} \mathrm{~A} \mathrm{~cm}^{-2}$ along the [11 0 ] direction (see the

6 black curve in Fig. 2b), which might result from slight phase separation of the (Ga0.94,Mn0.06)As

7 layer.

8 In the magnetisation switching behaviour shown in Fig. $2 \mathrm{~b}, J_{\mathrm{c}}$ is $8.2 \times 10^{5} \mathrm{~A} \mathrm{~cm}^{-2}$ at $40 \mathrm{~K}$ for

$9 \quad J / /[\overline{1} 10]$. By changing $J$ towards the [110] direction, $J_{\mathrm{c}}$ is found to be increased to $10.5 \times 10^{5} \mathrm{~A}$

$10 \mathrm{~cm}^{-2}$. This occurs because $H_{\mathrm{R}}$ is induced in the $\mathrm{Au} / \mathrm{Cr} / \mathrm{AlO}_{x} /(\mathrm{Ga}, \mathrm{Mn})$ As system by the breaking

11 of the structure inversion symmetry with the 40 -nm-thick $\mathrm{AlO}_{x}$ layer and the $\mathrm{Au} / \mathrm{Cr}$ electrode. As

12 shown in Fig. 1b, under $J / /[\overline{1} 10], H_{\mathrm{R}}$ is parallel to $H_{\mathrm{D}}$, which enhances the total effective field

$13 H_{\text {eff }}$ along the in-plane direction, where $H_{\text {eff }}=H_{\mathrm{D}}+H_{\mathrm{R}}$, and makes the spin component exert a

14 strong SOT on the magnetisation. In contrast, under $J / /$ [110], the direction of $H_{\mathrm{R}}$ is opposite to

15 that of $H_{\mathrm{D}}$, which suppresses $H_{\text {eff }}\left(H_{\text {eff }}=H_{\mathrm{D}}-H_{\mathrm{R}}\right)$ and weakens the SOT. Therefore, the

16 switching process is hindered by $H_{\mathrm{R}}$ when $J$ flows along the [110] direction, resulting in an

17 increase in $J_{\mathrm{c}}$. The aforementioned results indicate that the direction of the induced spin

18 component is always along $H_{\mathrm{D}}$ (see Fig. 1b). Hence, we can conclude that $H_{\mathrm{D}}$ is dominant during

19 the SOT switching in the $\mathrm{Au} / \mathrm{Cr} / \mathrm{AlO}_{x} /(\mathrm{Ga}, \mathrm{Mn}) \mathrm{As}$ system and that $H_{\mathrm{R}}$ can assist the switching 20 process for $J / /[\overline{1} 10]$ but hinders it for $J / /[110]$.

21

\section{Manipulation of SOT switching via a gate electric field}


To achieve manipulation of the SOT switching with an electric field, we applied a gate voltage $V_{\mathrm{g}}$ to the gate electrode shown in Fig. 1a. First, we measured the electrical characteristics of the crossbar device, where the source-drain current $I_{\mathrm{SD}}$ was measured as a function of the source-drain voltage $V_{\mathrm{SD}}$ at $40 \mathrm{~K}$ by applying various $V_{\mathrm{g}}$ of $0 \mathrm{~V}, \pm 1 \mathrm{~V}, \pm 5 \mathrm{~V}, \pm 10 \mathrm{~V}, \pm 15 \mathrm{~V}$ and \pm $20 \mathrm{~V}$, as shown in Fig. 3a. Here, $V_{\mathrm{SD}}$ ranging from $+0.1 \mathrm{~V}$ to $-0.1 \mathrm{~V}$ was applied with a step of $0.001 \mathrm{~V}$. The $I_{\mathrm{SD}}-V_{\mathrm{SD}}$ curves in Fig. 3a show an obvious ohmic character, meaning that ohmic contacts are formed in the crossbars. The contacts remained in good condition and were not destroyed during the measurements. By applying $V_{\mathrm{g}}$, we found that $I_{\mathrm{SD}}$ was varied, indicating that $I_{\mathrm{SD}}$ can be manipulated by $V_{\mathrm{g}}$. To more clearly understand the change in $I_{\mathrm{SD}}$ when applying different $V_{\mathrm{g}}, I_{\mathrm{SD}}$ modulation ratios at various $V_{\mathrm{g}}$ are plotted in Fig. $3 \mathrm{~b}$ based on the results shown in Fig. 3a. Here, the modulation ratio is defined by $\Delta I_{\mathrm{SD}} / I_{\mathrm{SD}}\left(V_{\mathrm{g}}=0\right) \times 100 \%$, where $\Delta I_{\mathrm{SD}}$ is the $I_{\mathrm{SD}}$ modulation defined by $\Delta I_{\mathrm{SD}}=I_{\mathrm{SD}}\left(V_{\mathrm{g}}\right)-I_{\mathrm{SD}}\left(V_{\mathrm{g}}=0\right)$. In Fig. 3b, an obvious $I_{\mathrm{SD}}$ change occurs that is modulated by $V_{\mathrm{g}}$. For $V_{\mathrm{g}}<0$, the $I_{\mathrm{SD}}$ modulation ratio is positive, indicating that the negative $V_{\mathrm{g}}$ increases the hole concentration in the $\left(\mathrm{Ga}_{0.94}, \mathrm{Mn}_{0.06}\right)$ As layer. In contrast, for $V_{\mathrm{g}}>0$, the ISD modulation ratio is negative, resulting from the decrease in the hole concentration in the (Ga0.94,Mn0.06)As thin film.

Next, SOT switching was induced by sweeping $J$ along the [110] direction with application of a $V_{\mathrm{g}}$ of $\pm 10 \mathrm{~V}$, as shown in Fig. 3c. From the results, the application of the positive $V_{\mathrm{g}}=+10$ $\mathrm{V}$ decreases $J_{\mathrm{c}}$ from $8.2 \times 10^{5}$ to $7.6 \times 10^{5} \mathrm{~A} \mathrm{~cm}^{-2}$, which means that the SOT switching efficiency is enhanced for $V_{\mathrm{g}}>0$. Meanwhile, for $V_{\mathrm{g}}=-10 \mathrm{~V}, J_{\mathrm{c}}$ increases to $8.7 \times 10^{5} \mathrm{~A} \mathrm{~cm}^{-2}$, indicating that the negative $V_{\mathrm{g}}$ hinders the switching process to some extent. Hence, by applying the electric field $\left(V_{\mathrm{g}}= \pm 10 \mathrm{~V}\right), J_{\mathrm{c}}$ can be efficiently modulated by $\sim 14.5 \%[=(8.7-7.6) / 7.6 \times 100 \%]$. Figure $3 \mathrm{~d}$ shows the manipulation of the SOT switching $(J / /[\overline{1} 10])$ at different $V_{\mathrm{g}}$ of $\pm 5 \mathrm{~V}, \pm 10 \mathrm{~V}, \pm 15$ 
$1 \mathrm{~V}$ and $\pm 20 \mathrm{~V}$ at $40 \mathrm{~K}$ (see Supplementary Note 3 in the Supplementary Information for details),

2 from which we can conclude that a positive $V_{\mathrm{g}}$ decreases $J_{\mathrm{c}}$ and that a negative $V_{\mathrm{g}}$ increases $J_{\mathrm{c}}$.

3 These results indicate that the switching behaviour is solidly reversibly modulated via the

4 electric field.

6 Mechanism of the electric field control of the SOT switching

7 The physical mechanism of the electric field modulation of the SOT switching in the

$8(\mathrm{Ga}, \mathrm{Mn})$ As layer can be attributed to the successful modulation of the interfacial electric field $E$.

9 As shown in Fig. 4a, when $V_{\mathrm{g}}$ is 0 , an internal electric field $E_{0}$ is generated because of the

10 existence of a thin depletion layer in the $\left(\mathrm{Ga}_{0.94}, \mathrm{Mn}_{0.06}\right)$ As layer in the vicinity of the $\mathrm{AlO}_{x}$ layer,

11 as shown in Fig. 4d. By applying a positive $V_{\mathrm{g}}$, the depletion is enhanced, as shown in Fig. 4e,

12 which generates an additional interfacial electric field $E_{1}$ that points in the same direction as $E_{0}$,

13 as shown in Fig. $4 \mathrm{~b}$. Therefore, the total $E$ becomes larger and contributes to a strong spin-orbit

14 interaction, which enhances $H_{\mathrm{R}}$ and $H_{\mathrm{D}}$. Then, $H_{\mathrm{R}}$ and $H_{\mathrm{D}}$ induce a large spin component along

15 the in-plane direction, which exerts a strong SOT on the magnetisation and enables a highly

16 efficient switching process with a small $J_{\text {c. }}$ When $V_{\mathrm{g}}$ is negative, holes accumulate near the

17 interface between the $\mathrm{AlO}_{x}$ and $(\mathrm{Ga}, \mathrm{Mn})$ As layers based on a capacitor model, which increases

18 the hole concentration near the $\mathrm{AlO}_{x}$ layer, as shown in Fig. 4f. Then, the direction of $E_{1}$ is

19 reversed, as shown in Fig. 4c, and the total $E$ is suppressed. The suppressed $E$ weakens $H_{\mathrm{R}}$ and

$20 H_{\mathrm{D}}$, resulting in a decrease in the strength of the SOT. Therefore, the magnetisation switching

21 process is suppressed, and $J_{\mathrm{c}}$ increases.

22 By changing the direction of $J$ from [110] to [110], the $J_{\mathrm{c}}$ modulation ratio obtained by

23 applying $V_{\mathrm{g}}$ decreases from $14.5 \%$ to $4.9 \%$ [ $\left.=(10.8-10.3) / 10.3 \times 100 \%\right]$, as shown in Fig. $5 \mathrm{a}$, 
1 where $J_{\mathrm{c}}$ is estimated to be $10.3 \times 10^{5} \mathrm{~A} \mathrm{~cm}^{-2}$ at $V_{\mathrm{g}}=+10 \mathrm{~V}$ and $10.8 \times 10^{5} \mathrm{~A} \mathrm{~cm}^{-2}$ at $V_{\mathrm{g}}=-10 \mathrm{~V}$.

2 The decrease in the $J_{\mathrm{c}}$ modulation ratio indicates that both $H_{\mathrm{D}}$ and $H_{\mathrm{R}}$ are influenced by $V_{\mathrm{g}}$ and

3 that the total manipulation effect is much stronger for $J / /[\overline{1} 10]$. This is reasonable because $H_{\mathrm{D}}$

4 and $H_{\mathrm{R}}$ point in the same direction for $J / /[\overline{1} 10]$, and the modulation effects are superimposed. In

5 contrast, when applying $J$ along the [110] axis, the directions of $H_{\mathrm{D}}$ and $H_{\mathrm{R}}$ are opposite, which

6 reduces the modulation effect obtained by applying the gate electric field because the total

7 enhancement or suppression effects of $H_{\mathrm{D}}$ and $H_{\mathrm{R}}$ cancel out. Hence, a small modulation ratio of

$8 J_{\mathrm{c}}$ is realized.

9 To check the influence of the modulation of $H_{\mathrm{c}}$ by the gate bias voltage, $R_{\mathrm{H}}$ is measured by 10 sweeping $H_{z}$ along the [001] direction at $V_{\mathrm{g}}=0$ and $\pm 10 \mathrm{~V}$. As shown in Fig. 5b, with changing

$11 V_{\mathrm{g}}, H_{\mathrm{c}}$ is nearly constant at approximately $300 \mathrm{Oe}$, indicating that $H_{\mathrm{c}}$ is not a determining factor

12 in the electric field control of the SOT switching. In addition, Fig. $5 \mathrm{~b}$ shows that $R_{\mathrm{H}}$ varies

13 between $\pm 0.587 \mathrm{k} \Omega$ when sweeping $H_{z}$ at $V_{\mathrm{g}}=+10 \mathrm{~V}$. At $V_{\mathrm{g}}=-10 \mathrm{~V}, R_{\mathrm{H}}$ varies between \pm 0.571

$14 \mathrm{k} \Omega$. Therefore, $R_{\mathrm{H}}$ can be slightly modulated by $V_{\mathrm{g}}$ with a modulation ratio of only $2.8 \%$ [=

$15(0.587-0.571) / 0.571 \times 100 \%)]$. Additionally, from the results shown in Fig. $3 \mathrm{a}, R$ is estimated to

16 be $193 \mathrm{k} \Omega$ at $V_{\mathrm{g}}=+10 \mathrm{~V}$ and $186 \mathrm{k} \Omega$ at $V_{\mathrm{g}}=-10 \mathrm{~V}$. In ferromagnets, $R_{\mathrm{H}}=R_{\mathrm{S}} M+R_{0} B$, where $R_{\mathrm{S}}$

17 is the anomalous Hall coefficient and is proportional to either $R$ or $R^{2}$, depending on the AHE

18 mechanism (skew or side-jump scattering) ${ }^{20} . M$ is the magnetisation, $R_{0}$ is the ordinary Hall

19 coefficient, and $B$ is the magnetic field. If $R_{\mathrm{S}}$ is proportional to $R$, then the saturation

20 magnetisation can be considered to be slightly modulated by $V_{\mathrm{g}}$ with a modulation ratio of only

$21-0.9 \%[=(0.587 / 193-0.571 / 186) /(0.571 / 186) \times 100 \%)]$. If $R_{\mathrm{S}}$ is proportional to $R^{2}$, then the

22 modulation ratio of the saturation magnetisation is $-4.5 \%\left[=\left(0.587 / 193^{2}-0.571 / 186^{2}\right) /\right.$

$\left.\left.23\left(0.571 / 186^{2}\right) \times 100 \%\right)\right]$. However, both values are much smaller than the modulation of $J_{\mathrm{c}}(\sim 14.5 \%$ 
1 for $J / /[\overline{1} 10])$. Therefore, the saturation magnetisation also does not have a significant influence

2 on the electric field control of the SOT switching process. To check the effect of the gate

3 modulation of $H_{\mathrm{an}}$, we changed the direction of the external magnetic field from [001] to [110]

4 (along the $y$ axis), where $H_{y}$ represents the magnetic field intensity (Fig. 5c). From the 5 comparison of $H_{\mathrm{c}}$ in Fig. $5 \mathrm{~b}\left(H_{\mathrm{c}}=300 \mathrm{Oe}\right)$ and Fig. $5 \mathrm{c}$ (coercivity $\left.H_{\mathrm{c}}{ }^{\prime}=7580 \mathrm{Oe}\right)$, a $2.3 \mathrm{deg}$ [= $6 \arcsin (300 / 7580)]$ misalignment of the magnetic field occurs due to the misalignment of the

7 magnet and/or the sample, resulting in an additional AHE signal. Therefore, an out-of-plane

8 component of the external magnetic field with a value of 12 Oe $(=300 \times \sin (2.3 \mathrm{deg}))$ exists

9 during the current-induced SOT switching, but it is negligibly small. Here, the three curves 10 shown in Fig. 5c can be found to overlap with each other, which means that $H_{\text {an }}$ is constant when 11 changing $V_{\mathrm{g}}$ and that the influence of the modulation of $H_{\mathrm{an}}$ can also be excluded as a 12 determining factor in the successful manipulation of the SOT switching via the electric field.

\section{Conclusions}

15 In this work, the manipulation of SOT switching via a gate electric field is achieved, which can be ascribed to the successful modulation of the interfacial electric field. By applying a

17 positive $V_{\mathrm{g}}$, the strength of the electric field is enhanced, which strengthens the total effective field in (Ga,Mn)As and assists the magnetisation switching. In contrast, a negative $V_{\mathrm{g}}$ results in a smaller effective field, which decreases the switching efficiency and increases $J_{\text {c. This finding }}$

20 provides an approach towards reversible modulation of the SOT switching in a single layer of a

21 spin-orbit ferromagnet via electric fields, which will advance the development of energy-

22 efficient gate-controlled spin-torque devices and help further the understanding of the switching 23 mechanism. 
Methods

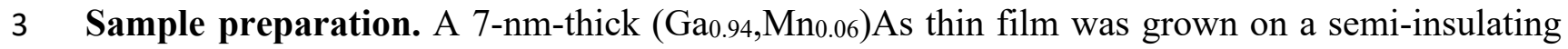

4 GaAs (001) substrate in an ultrahigh-vacuum MBE system. After removal of the surface oxide

5 layer of the GaAs substrate at $580{ }^{\circ} \mathrm{C}$, a 50 -nm-thick GaAs buffer layer was grown to obtain an

6 atomically smooth surface. After that, the substrate was cooled to $450{ }^{\circ} \mathrm{C}$, and a 500 -nm-thick

7 In ${ }_{0.3} \mathrm{Ga} 0.7 \mathrm{As}$ layer was grown to induce a tensile strain in the (Ga0.94, $\left.\mathrm{Mn}_{0.06}\right)$ As layer, giving rise

8 to PMA. Then, the sample was cooled to approximately $290{ }^{\circ} \mathrm{C}$ for growth of the 7 -nm-thick

9 (Ga0.94,Mn0.06)As layer. The growth process was monitored in situ by means of reflection high10 energy electron diffraction. The Curie temperature $T_{\mathrm{C}}$ of the $(\mathrm{Ga} 0.94, \mathrm{Mn} 0.06)$ As thin film was

11 estimated to be $75 \mathrm{~K}$. After the device fabrication process, the $T_{\mathrm{C}}$ increased to $112 \mathrm{~K}$, which

12 might be caused by the annealing process resulting from the increase in the sample temperature

13 during the device fabrication process (see Supplementary Note 2 in the Supplementary 14 Information for details).

15

16 Transport measurements. For the SOT measurements, a Keithley 2636A was used as the 17 current source for applying a direct current, and a Keithley 2400 was used for applying a gate 18 voltage. The Hall voltage was measured with another Keithley 2400. The measurements were 19 carried out at $40 \mathrm{~K}$.

20

\section{Data availability}

23 Acknowledgements 
1 This work was partly supported by Grants-in-Aid for Scientific Research (No. 16H02095, No.

2 18H03860, No. 20H05650), the CREST program of the Japan Science and Technology Agency

3 (JPMJCR1777), the Spintronics Research Network of Japan (Spin-RNJ), and the China

$4 \quad$ Scholarship Council (No. 201706210086).

5

6 Author Contributions

7 Sample preparation: M. J. and H. A.; measurements: M. J.; data analysis: M. J., S. O. and M. T.;

8 writing and project planning: M. J., S. O. and M. T.

9

10 Additional information

11 The authors declare no competing financial interests. Reprints and permission information is

12 available online at http://npg.nature.com/reprintsandpermissions/. Correspondence and requests

13 for materials should be addressed to M. T.

14

15 Supplementary information is available for this paper at URL. 
2 1. Liu, L., Lee, O. J., Gudmundsen, T. J., Ralph, D. C., and Buhrman, R. A. Current-induced

\section{References} switching of perpendicularly magnetized magnetic layers using spin torque from the spin Hall effect. Phys. Rev. Lett. 109, 096602 (2012).

2. Khang, N. H. D., Ueda, Y., Hai, P. N. A conductive topological insulator with large spin Hall effect for ultralow power spin-orbit torque switching. Nat. Mater. 17, 808-813 (2018).

3. Liu, R. H., Lim, W., Urazhdin, S. Control of current-induced spin-orbit effects in a ferromagnetic heterostructure by electric field. Phys. Rev. B 89, 220409 (2014).

4. Emori, S., Bauer, U., Woo, S., Beach, G. S. D. Large voltage-induced modification of spin-orbit torques in $\mathrm{Pt} / \mathrm{Co} / \mathrm{GdO}_{x}$. Appl. Phys. Lett. 105, 222401 (2014).

5. Mishra, R. et al. Electric-field control of spin accumulation direction for spin-orbit torques. Nat Commun. 10, 1-8 (2019).

6. Yan, Y. et al. Strong electrical manipulation of spin-orbit torque in ferromagnetic heterostructures. Adv. Electron. Mater. 2, 1600219 (2016).

7. Endo, M., Matsukura, F., Ohno, H. Current induced effective magnetic field and magnetization reversal in uniaxial anisotropy (Ga,Mn)As. Appl. Phys. Lett. 97, 222501 (2010).

8. Jiang, M. et al. Efficient full spin-orbit torque switching in a single layer of a perpendicularly magnetized single-crystalline ferromagnet. Nat. Commun. 10, 259019 (2019).

9. Chernyshov, A. et al. Evidence for reversible control of magnetization in a ferromagnetic material by means of spin-orbit magnetic field. Nat. Phys. 5, 656-659 (2009).

10. Fan, Y. et al. Electric-field control of spin-orbit torque in a magnetically doped topological insulator. Nat. Nanotechnol. 11, 352-359 (2016). 
1 11. Jiang, M., Asahara, H., Sato, S., Ohya, S., and Tanaka, M. Artificial suppression of the field-

2 like term and ultra-efficient magnetisation switching in a spin-orbit ferromagnet. Nat. $3 \quad$ Electron. in press.

4 12. Kou, X. et al. Manipulating surface-related ferromagnetism in modulation-doped topological 5 insulators. Nano. Lett. 13, 4587-4593 (2013).

6 13. Kou, X. et al. Interplay between different magnetisms in Cr-doped topological insulators. Acs $7 \quad$ Nano. 7, 9205-9212 (2013).

8 14. Chen, L. et al. Electric-field control of interfacial spin-orbit fields. Nat. Electron. 1, 350-355 $9 \quad(2018)$.

10 15. Lv, W. et al. Electric-field control of spin-orbit torques in $\mathrm{WS}_{2} /$ Permalloy bilayers. Acs Appl. $11 \quad$ Mater. Interfaces 10, 2843-2849 (2018).

12 16. Fang, D. et al. Spin-orbit-driven ferromagnetic resonance. Nat. Nanotechnol. 6, 413-417 13 (2011).

14 17. Dresselhaus, G. Spin-orbit coupling effects in zinc blende structures. Phys. Rev. 100, 580 15 (1955).

16 18. Winkler, R. Spin-orbit coupling effects in two-dimensional electron and hole systems. 17 Springer Tr. Mod. Phys. 191, 1-8 (2003).

18 19. Manchon, A., Koo, H. C., Nitta, J., Frolov, S. M., and Duine, R. A. New perspectives for 19 Rashba spin-orbit coupling. Nat. Mater. 14, 871-882 (2015).

20 20. Omiya, T. et al. Magnetotransport properties of (Ga, Mn) As investigated at low temperature 21 and high magnetic field. Physica E Low Dimens. Syst. Nanostruct. 7, 976-980 (2000). 
a

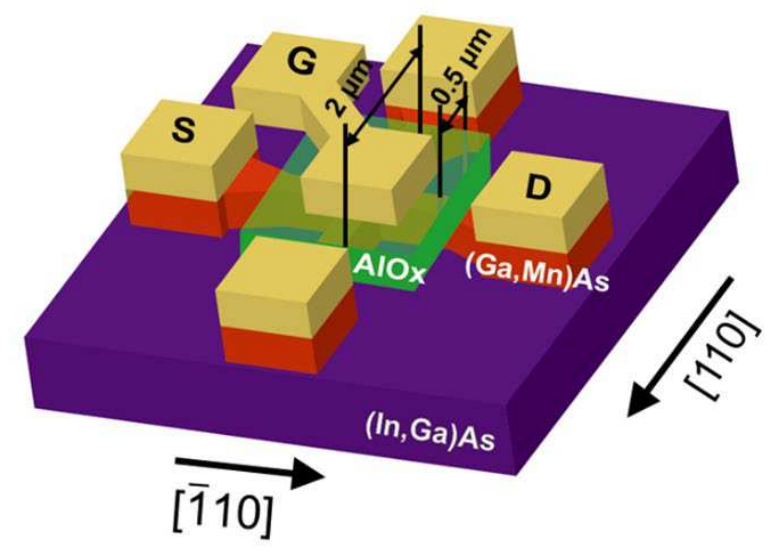

b

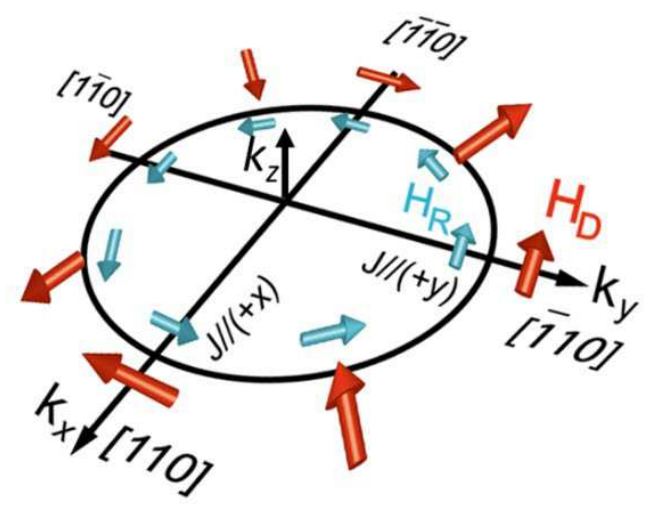

Figure 1 Schematic illustrations of the device structure and the effective magnetic field. a,

3 Schematic of the SOT device structure. The channel width of the crossbar is $0.5 \mu \mathrm{m}$, and the

4 channel length is $2 \mu \mathrm{m}$. The yellow parts are the capping layers on the electrodes consisting of $5 \mathrm{Au}(100 \mathrm{~nm}) / \mathrm{Cr}(5 \mathrm{~nm})$, which also work as heat sinks. The current $J$ flows in the 6 (Ga0.94,Mn0.06)As layer with perpendicular magnetisation from the source electrode (S) to the 7 drain electrode (D), and the Hall resistance $R_{\mathrm{H}}$ is obtained by measuring the Hall voltage $V_{\mathrm{H}}$ 8 along the [110] direction orthogonal to the S-D direction. An electric field is applied by the gate

9 voltage $V_{\mathrm{g}}$. b, Dresselhaus-like (red) and Rashba-like (light blue) effective magnetic fields $\left(H_{\mathrm{D}}\right.$ 10 and $H_{\mathrm{R}}$, respectively) for hole momenta along different crystallographic directions in the tensile11 strained $\left(\mathrm{Ga}_{0.94}, \mathrm{Mn}_{0.06}\right)$ As thin film. $\left(k_{x}, k_{y}, k_{z}\right)$ is the wave vector of holes. 
a

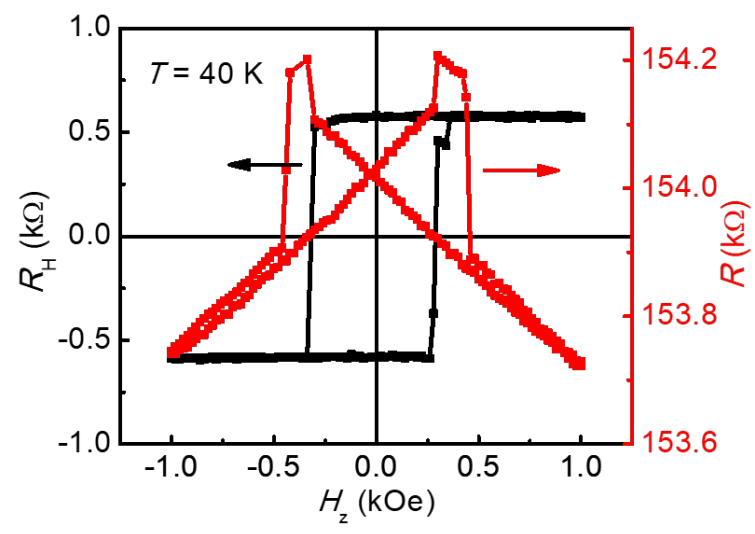

C

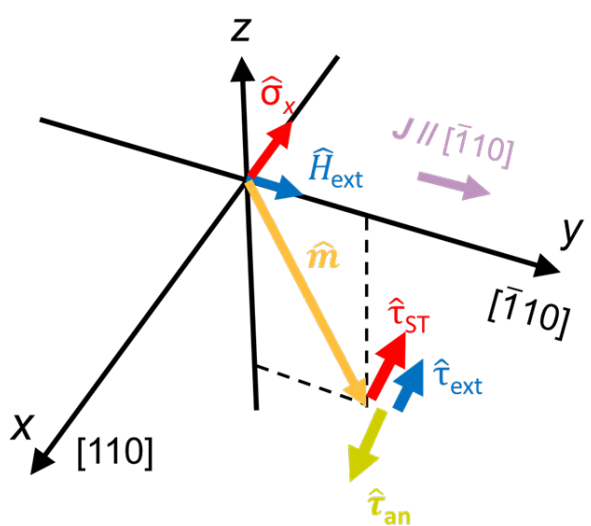

b

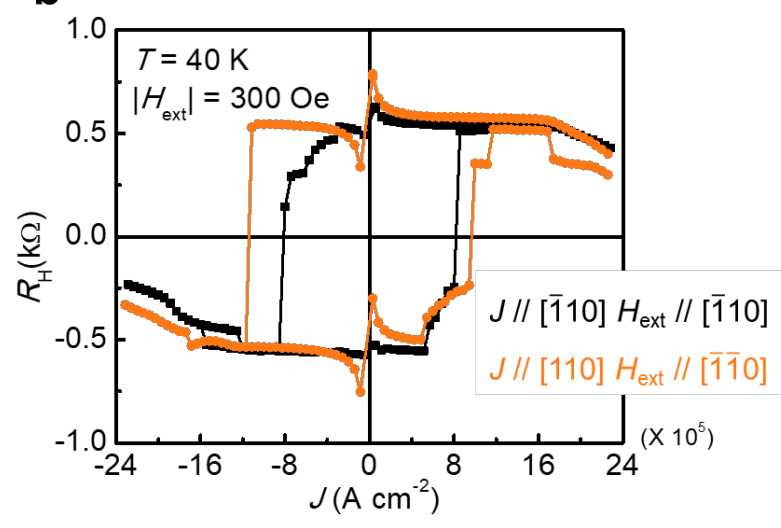

d

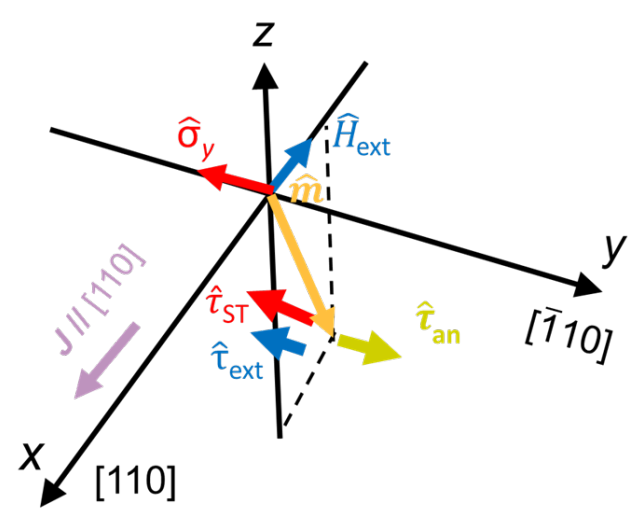

1

2 Figure 2 Characterisation of the AHE and AMR effect, and SOT switching. a,

3 Perpendicular magnetic field $H_{\mathrm{z}}$ dependence of the Hall resistance $R_{\mathrm{H}}$ and resistance $R$ of 4 (Ga0.94,Mn 0.06$)$ As at 40 K. b, Field-assisted SOT switching with $J / /[\overline{1} 10]$ and [110] in a 7-nm-

5 thick $\left(\mathrm{Ga}_{0.94}, \mathrm{Mn}_{0.06}\right)$ As thin film at $40 \mathrm{~K}$, where the magnitude of $H_{\mathrm{ext}}$ is 300 Oe. c, d, 6 Illustrations of the torques exerted by the spin component $\left(\hat{\boldsymbol{\tau}}_{\mathrm{ST}}\right)$, the external field $\left(\hat{\boldsymbol{\tau}}_{\text {ext }}\right)$ and the 7 anisotropy field $\left(\hat{\boldsymbol{\tau}}_{\mathrm{an}}\right)$ with $J>0$ for $J / /[\overline{1} 10], H_{\mathrm{ext}} / /[\overline{1} 10]$ (c) and $J / /[110], H_{\mathrm{ext}} / /[\overline{1} \overline{1} 0](\mathbf{d}) . \widehat{\mathbf{m}}$ 8 is in the $y-z$ plane (c) and $x-z$ plane (d). (Source data are provided as a Source Data file.) 
a

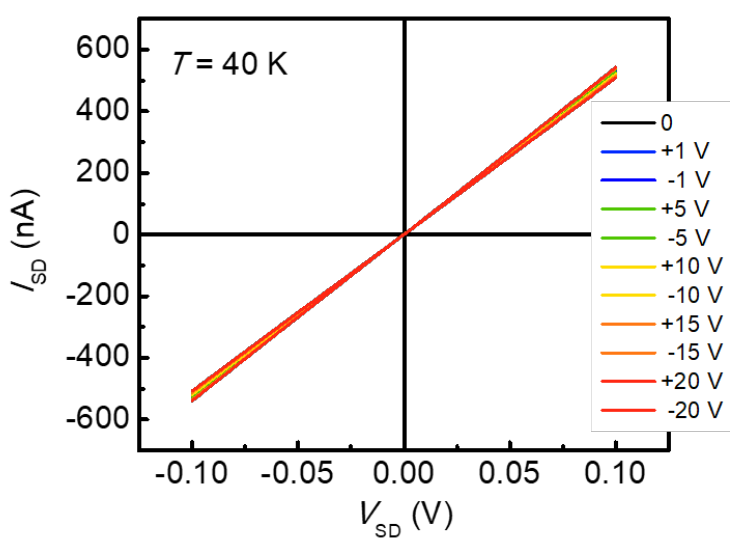

C

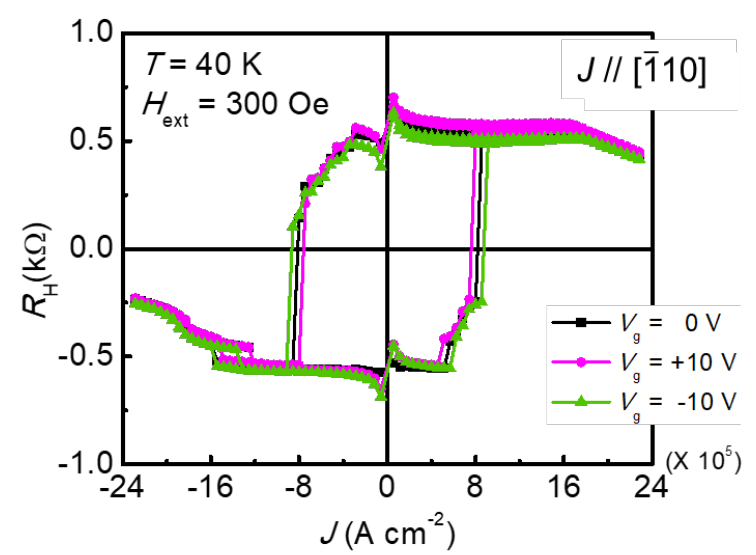

b

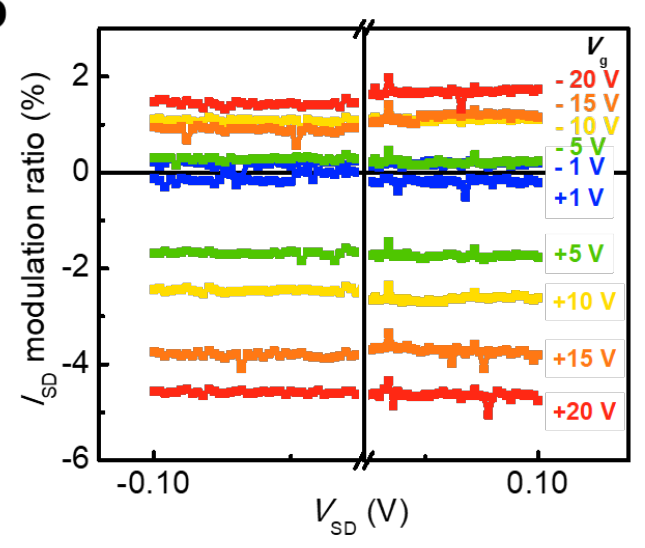

d

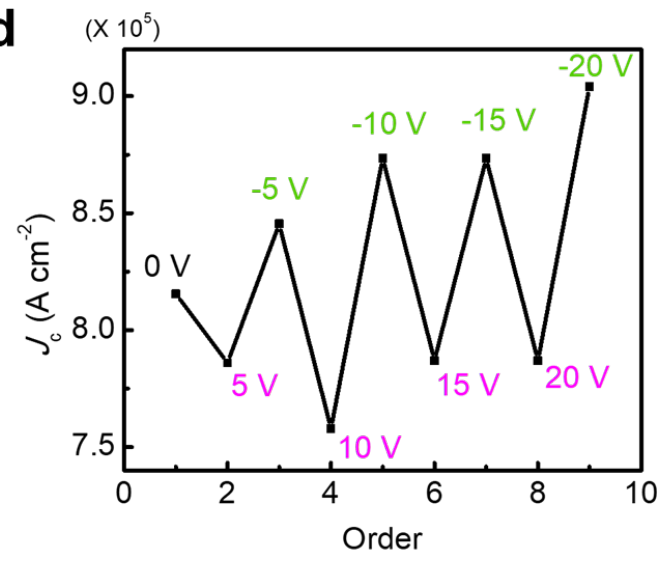

Figure $3 \mid V_{\mathrm{g}}$ dependence of the $I_{\mathrm{SD}}$ modulation and SOT switching with $J / /[\overline{1} 10]$. a,

$3 \quad I \mathrm{SD}-V \mathrm{SD}$ characteristics and $\mathbf{b}, I_{\mathrm{SD}}$ modulation ratio with the application of various gate voltages

$4 V_{\mathrm{g}}$ of $0 \mathrm{~V}, \pm 1 \mathrm{~V}, \pm 5 \mathrm{~V}, \pm 10 \mathrm{~V}, \pm 15 \mathrm{~V}$ and $\pm 20 \mathrm{~V}$ at $40 \mathrm{~K}$. $V$ SD ranging from $+0.1 \mathrm{~V}$ to $-0.1 \mathrm{~V}$

5 was applied with a step of 0.001 V. c, Field-assisted SOT switching by applying $V_{\mathrm{g}}$ of 0 and \pm 10

6 V. d, Plot of $J_{\mathrm{c}}$ obtained with various $V_{\mathrm{g}}$ in a $7-\mathrm{nm}$-thick $\left(\mathrm{Ga}_{0.94}, \mathrm{Mn}_{0.06}\right)$ As thin film at $40 \mathrm{~K}$.

7 Here, $J / /[\overline{1} 10]$ and $H_{\text {ext }}=300$ Oe. In this measurement, we changed $V_{\mathrm{g}}$ in the order of $0 \mathrm{~V} \rightarrow+5$

$8 \mathrm{~V} \rightarrow-5 \mathrm{~V} \rightarrow+10 \mathrm{~V} \rightarrow-10 \mathrm{~V} \rightarrow+15 \mathrm{~V} \rightarrow-15 \mathrm{~V} \rightarrow+20 \mathrm{~V} \rightarrow-20 \mathrm{~V}$. (Source data are

9 provided as a Source Data file.) 
a $V_{g}=0 \mathrm{~V}$

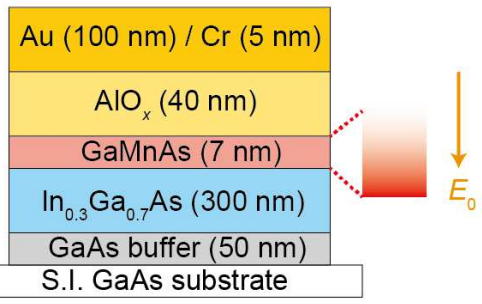

d

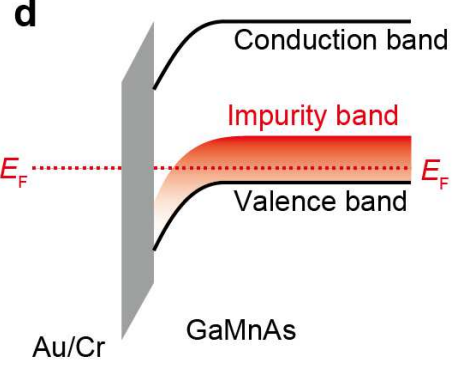

b $V_{g}>0 V$

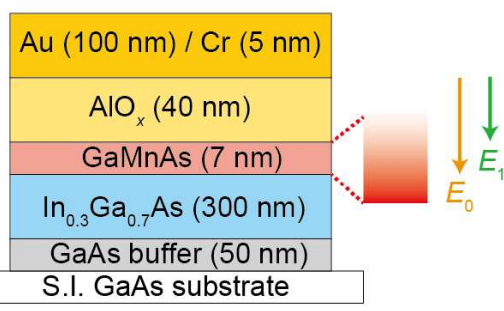

e

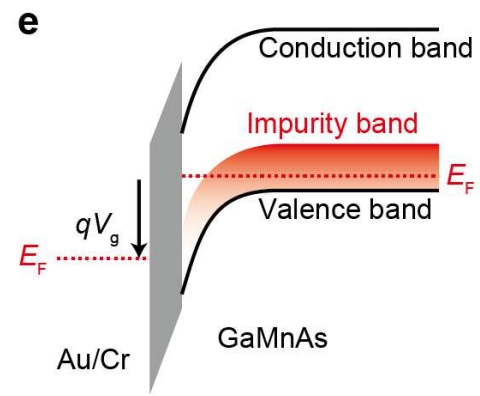

C $V_{\mathrm{g}}<0 \mathrm{~V}$

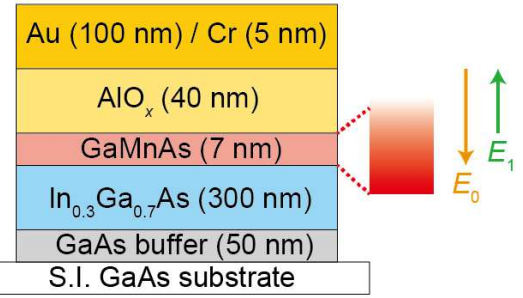

f

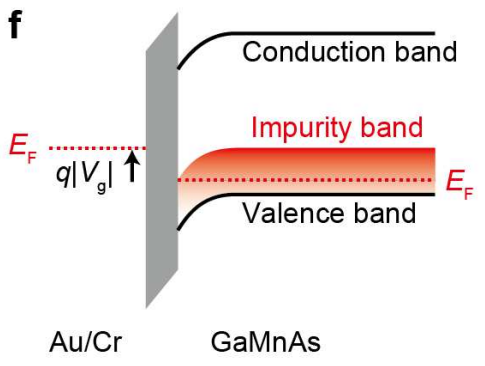

Figure 4 | Schematic illustrations of the interfacial electric field and the band structure.

3 Schematic illustration of the gate voltage modulation of the interfacial electric field under $\mathbf{a}, V_{\mathrm{g}}=$

$40 \mathrm{~V}, \mathbf{b}, V_{\mathrm{g}}>0 \mathrm{~V}$, and $\mathbf{c}, V_{\mathrm{g}}<0 \mathrm{~V}$. Schematic illustration of the band structure of

$5 \mathrm{Au} / \mathrm{Cr} / \mathrm{AlO}_{x} /(\mathrm{Ga}, \mathrm{Mn})$ As under $\mathbf{d}, V_{\mathrm{g}}=0 \mathrm{~V}, \mathbf{e}, V_{\mathrm{g}}>0 \mathrm{~V}$, and f, $V_{\mathrm{g}}<0 \mathrm{~V}$. 
a
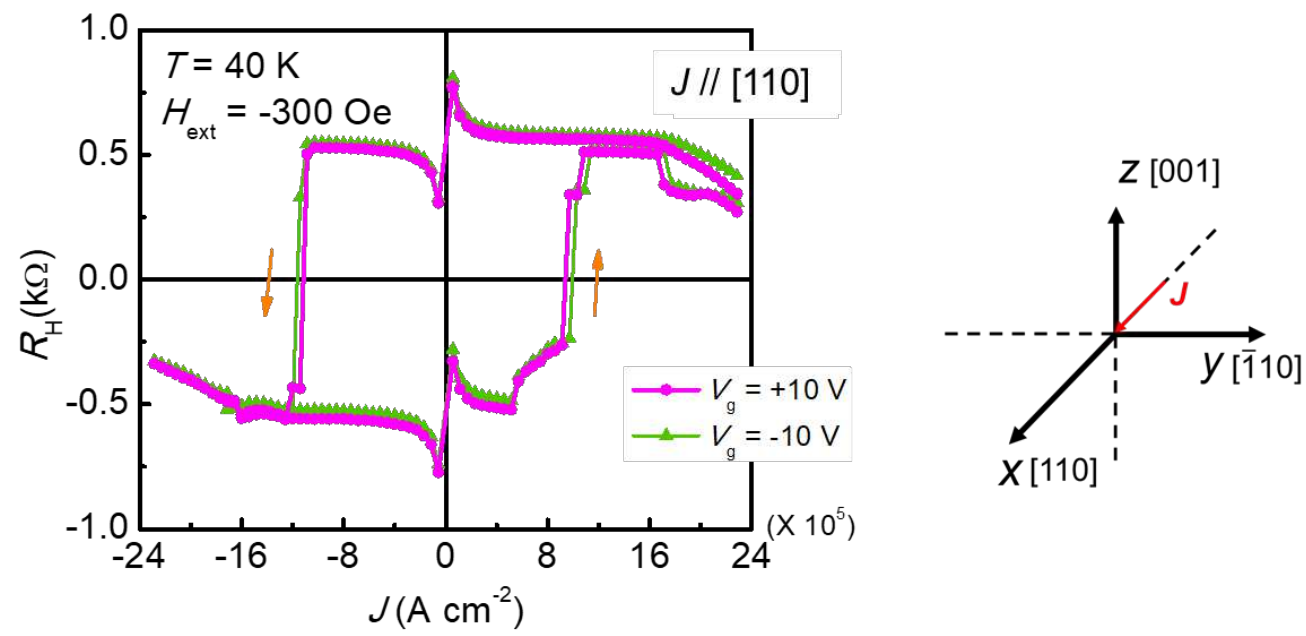

b

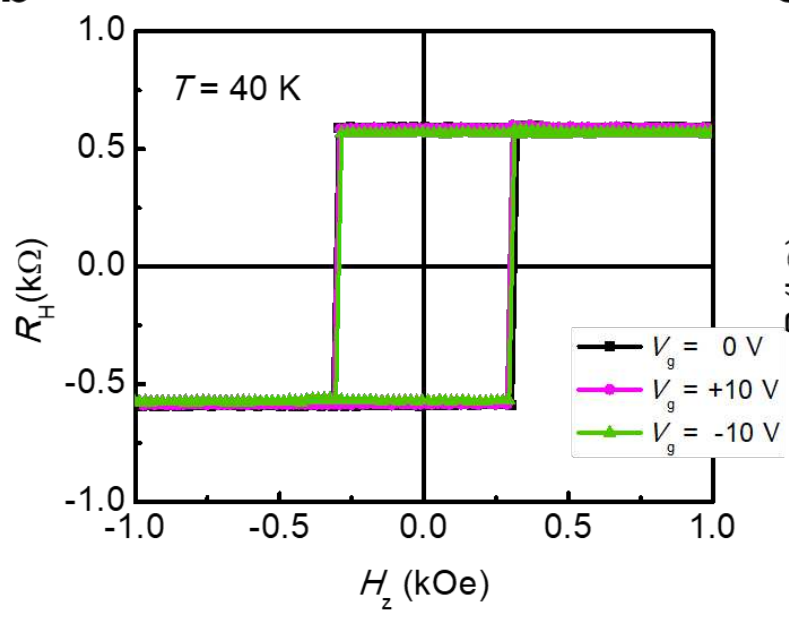

C

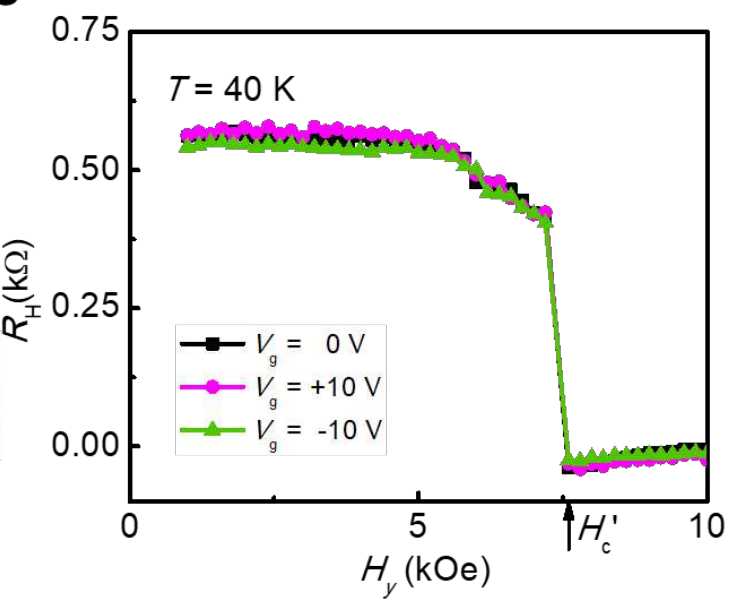

Figure $5 \mid V_{\mathrm{g}}$ dependence of SOT switching with $J / /[110], \boldsymbol{H}_{\mathrm{c}}$, and $\boldsymbol{H}_{\mathrm{an}}$. a, Field-assisted SOT

3 switching with $V_{\mathrm{g}}$ of 0 and $\pm 10 \mathrm{~V}$ in a 7 -nm-thick (Ga0.94,Mno.06)As thin film at $40 \mathrm{~K}$. Here, $J / /$

4 [110] and $H_{\mathrm{ext}}=-300$ Oe. b, Out-of-plane magnetic field $H_{z}$ dependence and c, in-plane

5 magnetic field $H_{y}$ dependence of the Hall resistance $R_{\mathrm{H}}$ of $(\mathrm{Ga} 0.94, \mathrm{Mn} 0.06)$ As with $V_{\mathrm{g}}$ of 0 and \pm 10

$6 \mathrm{~V}$ at $40 \mathrm{~K}$. The inset of (a) shows the coordinate relationship. (Source data are provided as a

7 Source Data file.) 
a

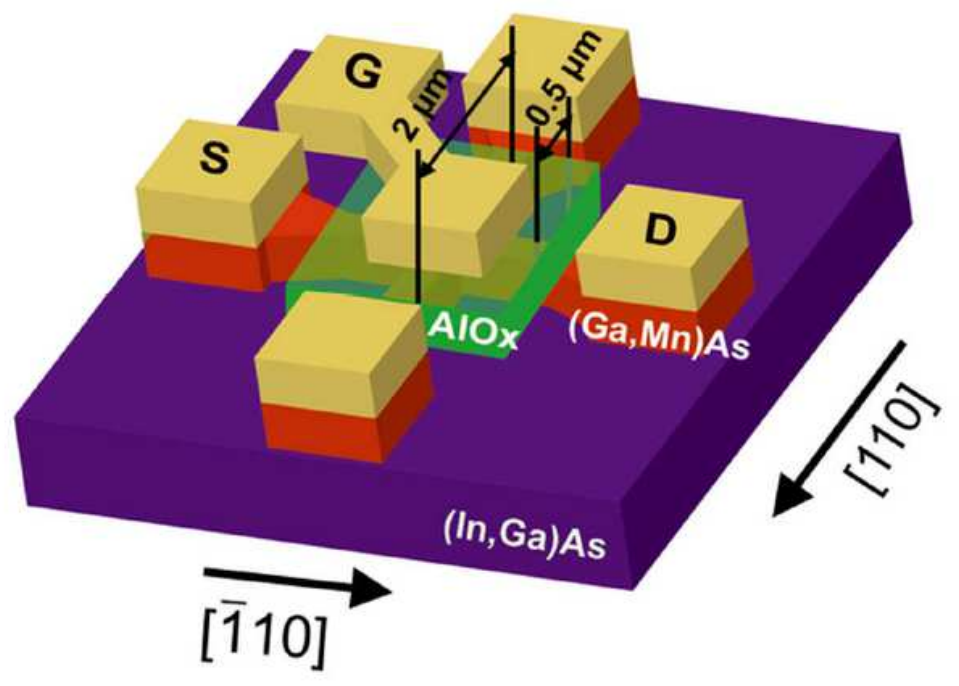

b

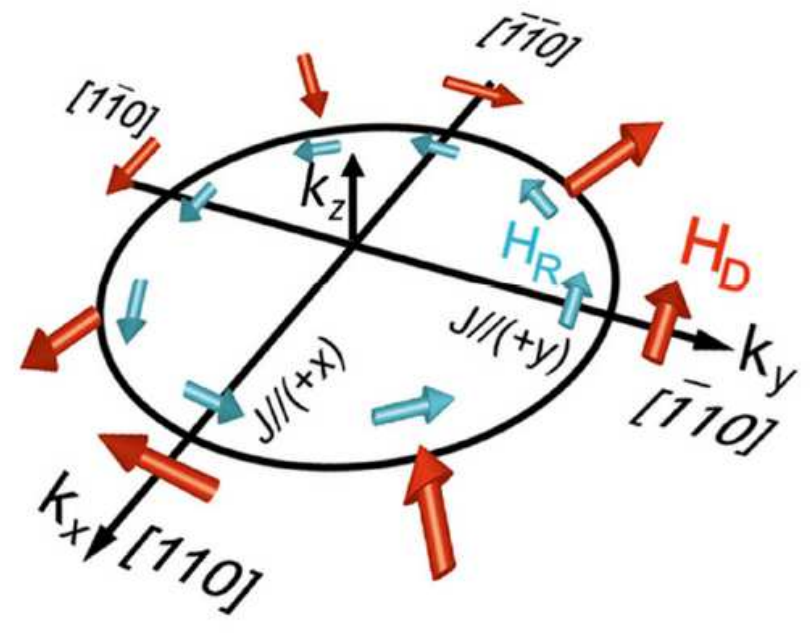

Figure 1

Schematic illustrations of the device structure and the effective magnetic field. a, Schematic of the SOT device structure. The channel width of the crossbar is $0.5 \mu \mathrm{m}$, and the channel length is $2 \mu \mathrm{m}$. The yellow parts are the capping layers on the electrodes consisting of $\mathrm{Au}(100 \mathrm{~nm}) / \mathrm{Cr}(5 \mathrm{~nm})$, which also work as heat sinks. The current $\mathrm{J}$ flows in the (Ga0.94,Mn0.06)As layer with perpendicular magnetisation from the source electrode (S) to the drain electrode (D), and the Hall resistance $\mathrm{RH}$ is obtained by measuring the Hall voltage $\mathrm{VH}$ along the [110] direction orthogonal to the S-D direction. An electric field is applied by the gate voltage Vg. b, Dresselhaus-like (red) and Rashba-like (light blue) effective magnetic fields (HD and $\mathrm{HR}$, respectively) for hole momenta along different crystallographic directions in the tensile-strained

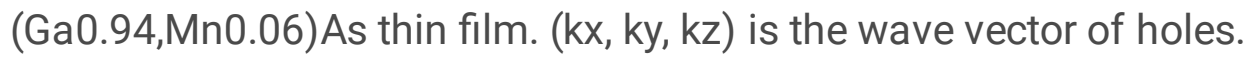


a

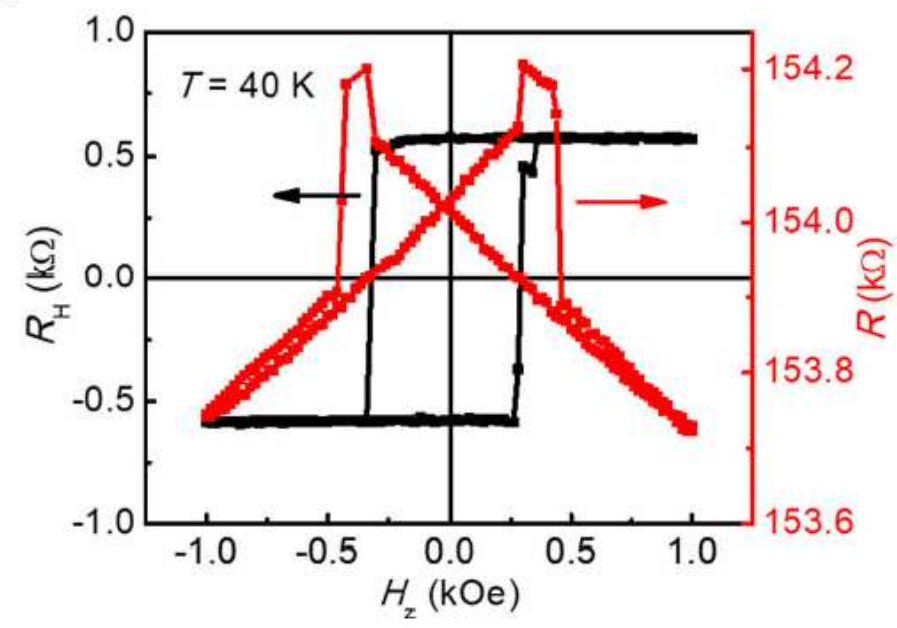

C

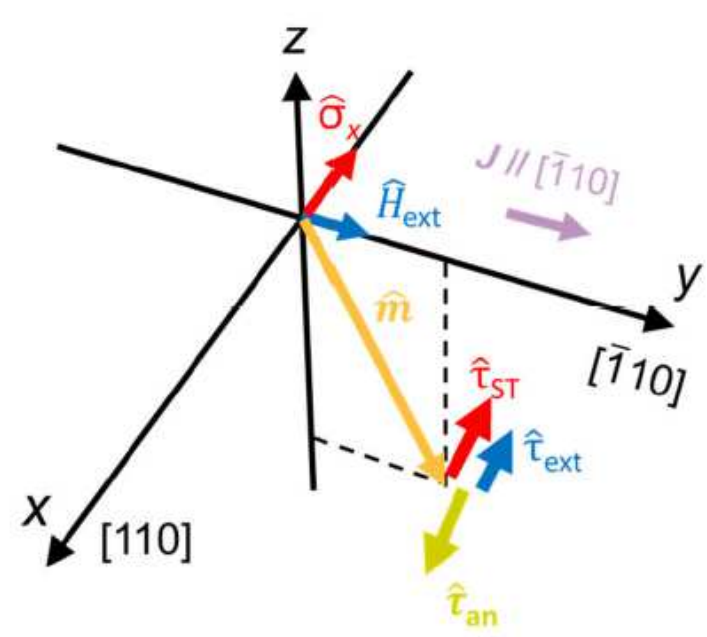

b

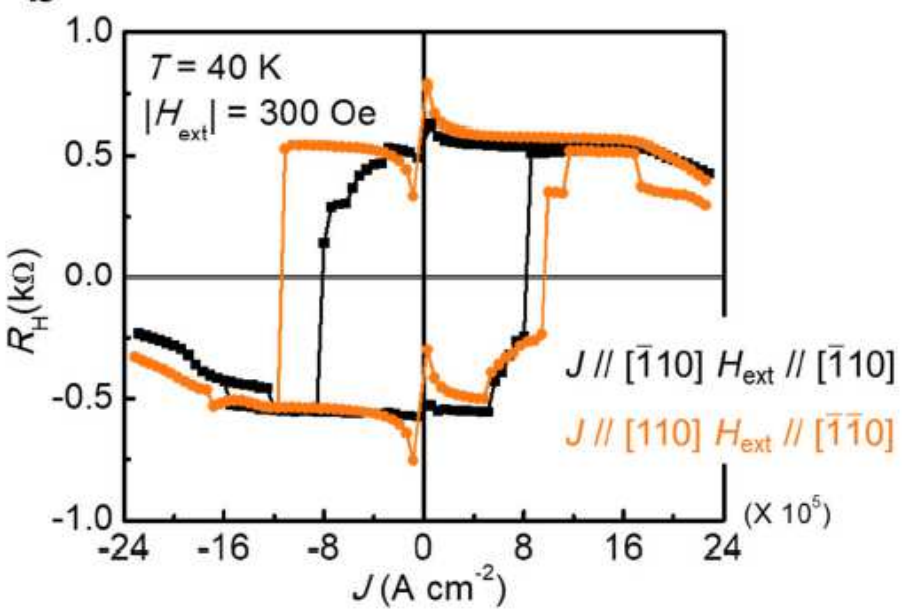

d

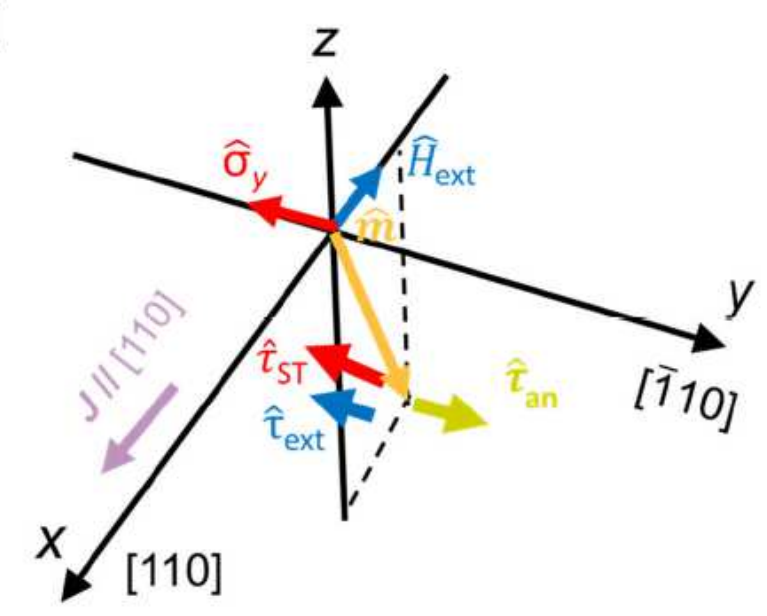

Figure 2

Characterisation of the AHE and AMR effect, and SOT switching. a, Perpendicular magnetic field $\mathrm{Hz}$ dependence of the Hall resistance RH and resistance R of (Ga0.94,Mn0.06)As at $40 \mathrm{~K}$. b, Field-assisted SOT switching with $\mathrm{J} / /\left[" 1 " \|^{\prime \prime} 10 "\right]$ and [110] in a 7-nm-thick (Ga0.94,Mn0.06)As thin film at $40 \mathrm{~K}$, where the magnitude of Hext is 300 Oe. c, d, lllustrations of the torques exerted by the spin component (" $\tau$ "

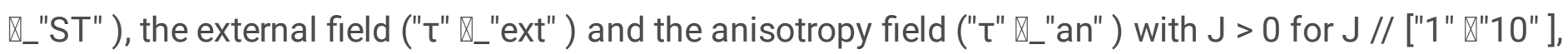

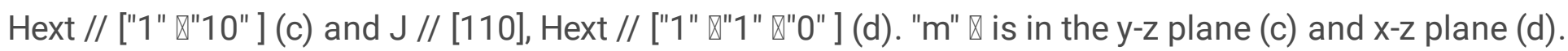
(Source data are provided as a Source Data file.) 
a

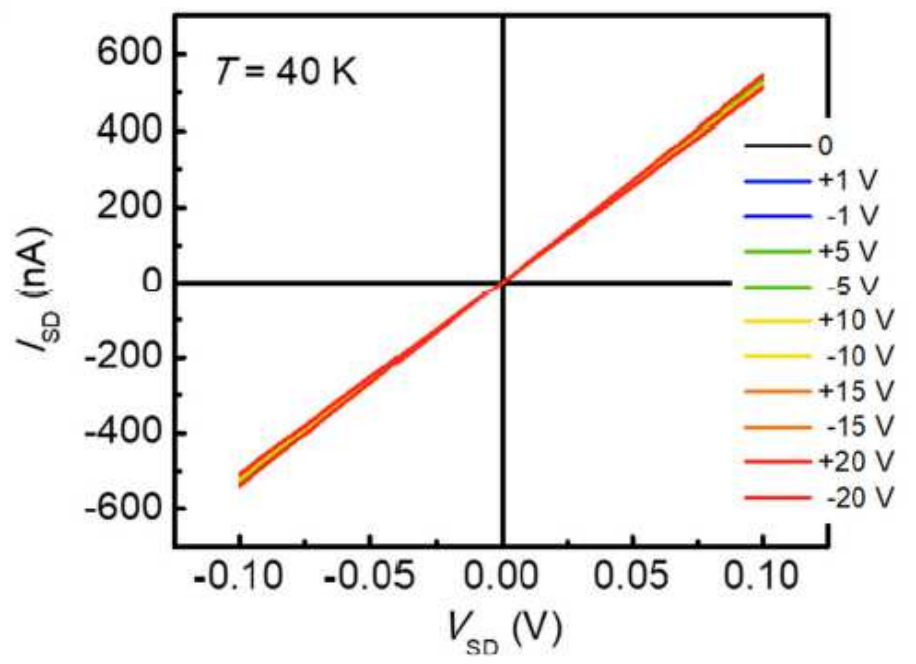

C

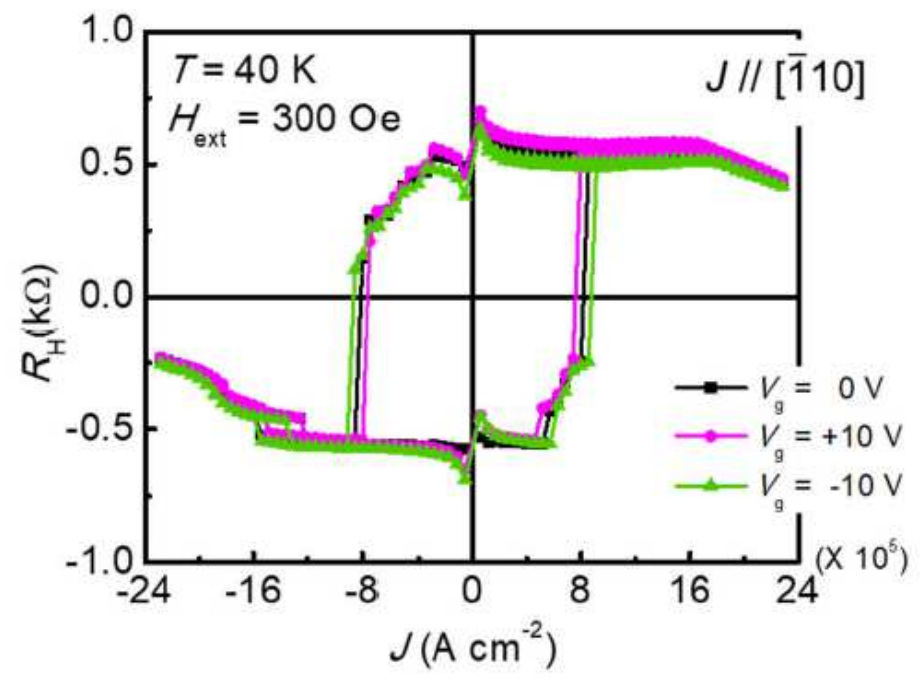

b

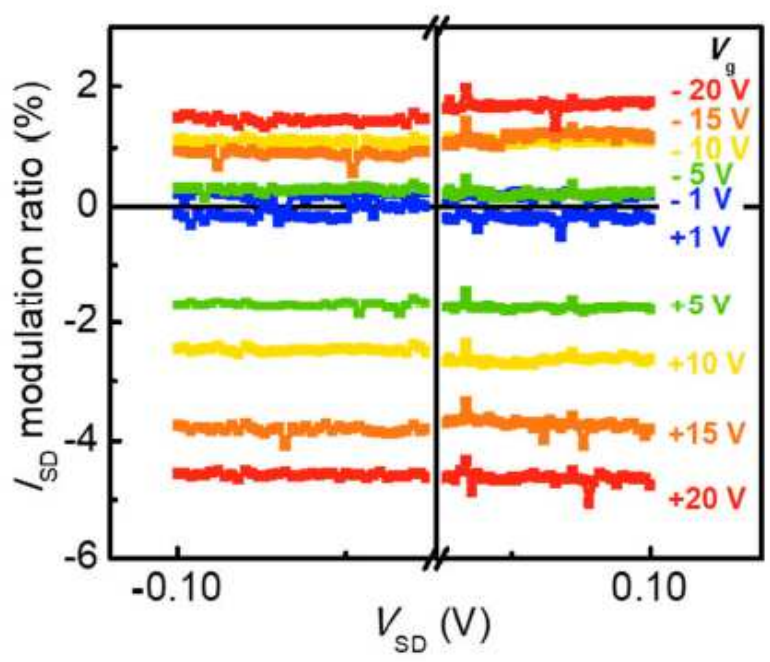

d

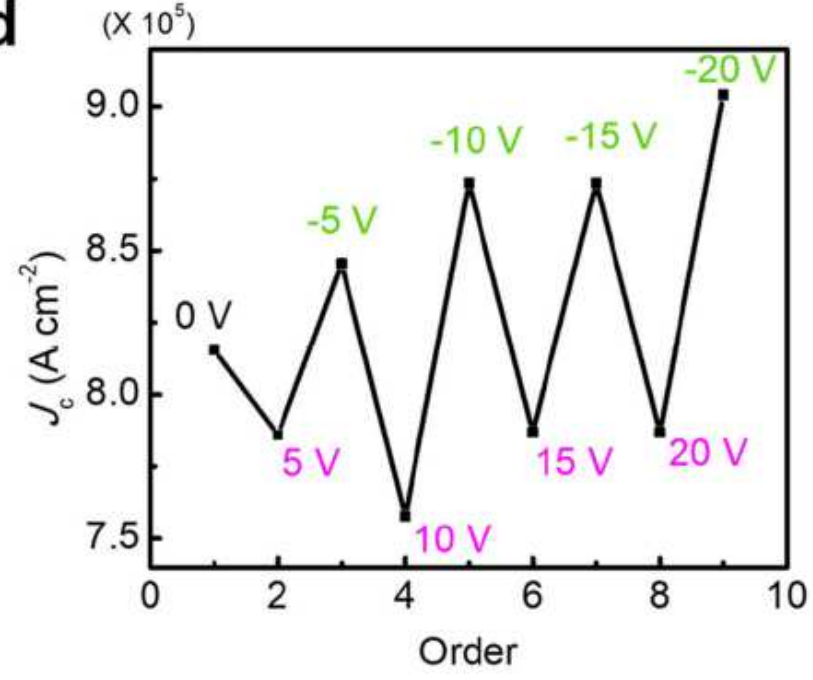

Figure 3

Vg dependence of the ISD modulation and SOT switching with $\mathrm{J} / /$ ["1" $"$ "10"]. a, ISD-VSD characteristics and $\mathrm{b}$, ISD modulation ratio with the application of various gate voltages $\mathrm{Vg}$ of $0 \mathrm{~V}, \pm 1 \mathrm{~V}, \pm 5 \mathrm{~V}, \pm 10 \mathrm{~V}, \pm 15$ $\mathrm{V}$ and $\pm 20 \mathrm{~V}$ at $40 \mathrm{~K}$. VSD ranging from $+0.1 \mathrm{~V}$ to $-0.1 \mathrm{~V}$ was applied with a step of $0.001 \mathrm{~V}$. c, Fieldassisted SOT switching by applying $\mathrm{Vg}$ of 0 and $\pm 10 \mathrm{~V}$. d, Plot of Jc obtained with various $\mathrm{Vg}$ in a 7-nmthick (Ga0.94,Mn0.06)As thin film at $40 \mathrm{~K}$. Here, $\mathrm{J} / /\left[" 1 " \nabla^{\prime \prime} 10 "\right]$ and Hext $=300 \mathrm{Oe}$. In this measurement, we changed $V g$ in the order of $0 \vee \nabla+5 V \nabla-5 V \nabla+10 \vee \nabla-10 \vee \nabla+15 \vee \nabla-15 \vee \nabla+20 \vee \nabla-20 \mathrm{~V}$. (Source data are provided as a Source Data file.) 


$$
\text { a } V_{g}=0 V
$$
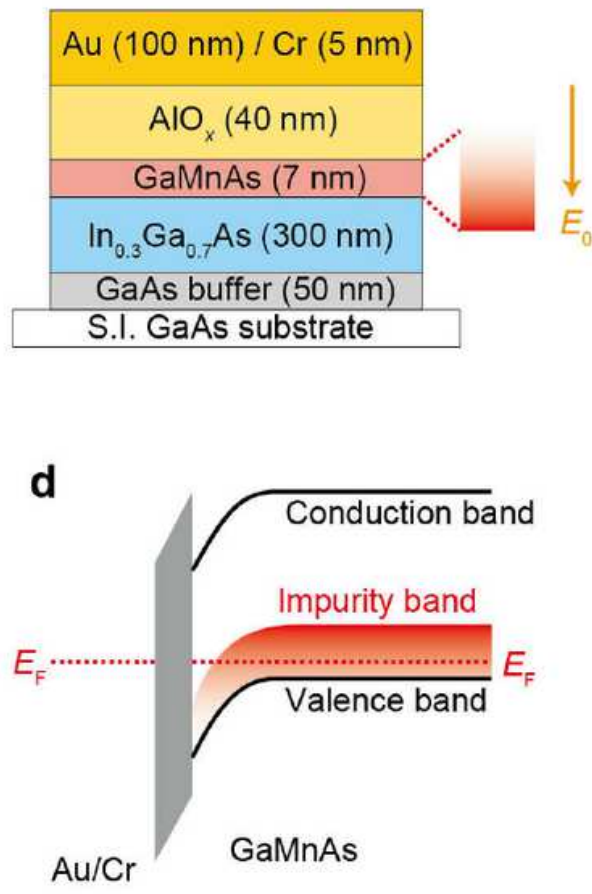
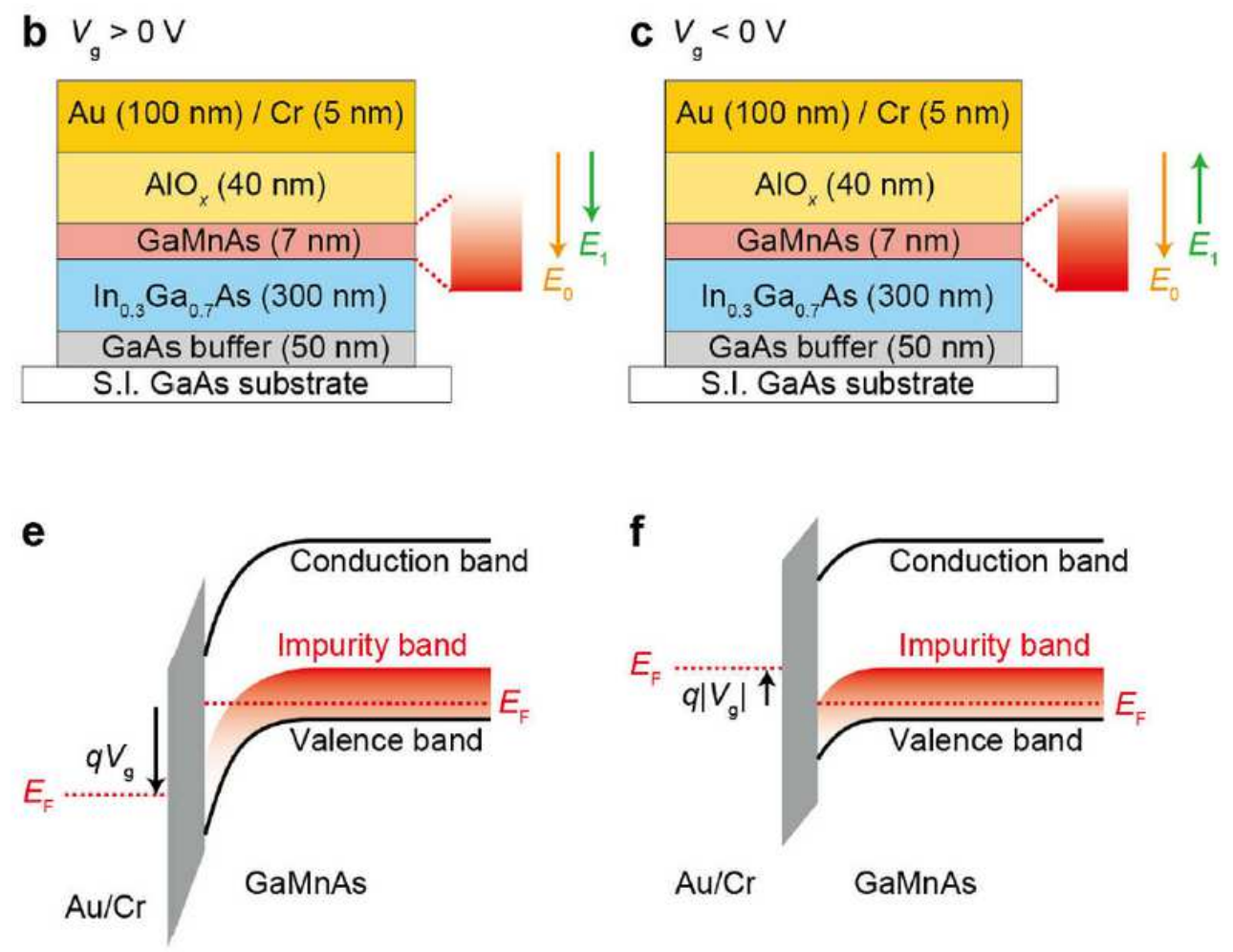

\section{Figure 4}

Schematic illustrations of the interfacial electric field and the band structure. Schematic illustration of the gate voltage modulation of the interfacial electric field under $a, V g=0 \mathrm{~V}, \mathrm{~b}, \mathrm{Vg}>0 \mathrm{~V}$, and $\mathrm{c}, \mathrm{Vg}<0 \mathrm{~V}$.

Schematic illustration of the band structure of $\mathrm{Au} / \mathrm{Cr} / \mathrm{AlOx} /(\mathrm{Ga}, \mathrm{Mn}) \mathrm{As}$ under $\mathrm{d}, \mathrm{Vg}=0 \mathrm{~V}, \mathrm{e}, \mathrm{Vg}>0 \mathrm{~V}$, and $\mathrm{f}$, $\mathrm{Vg}<0 \mathrm{~V}$. 

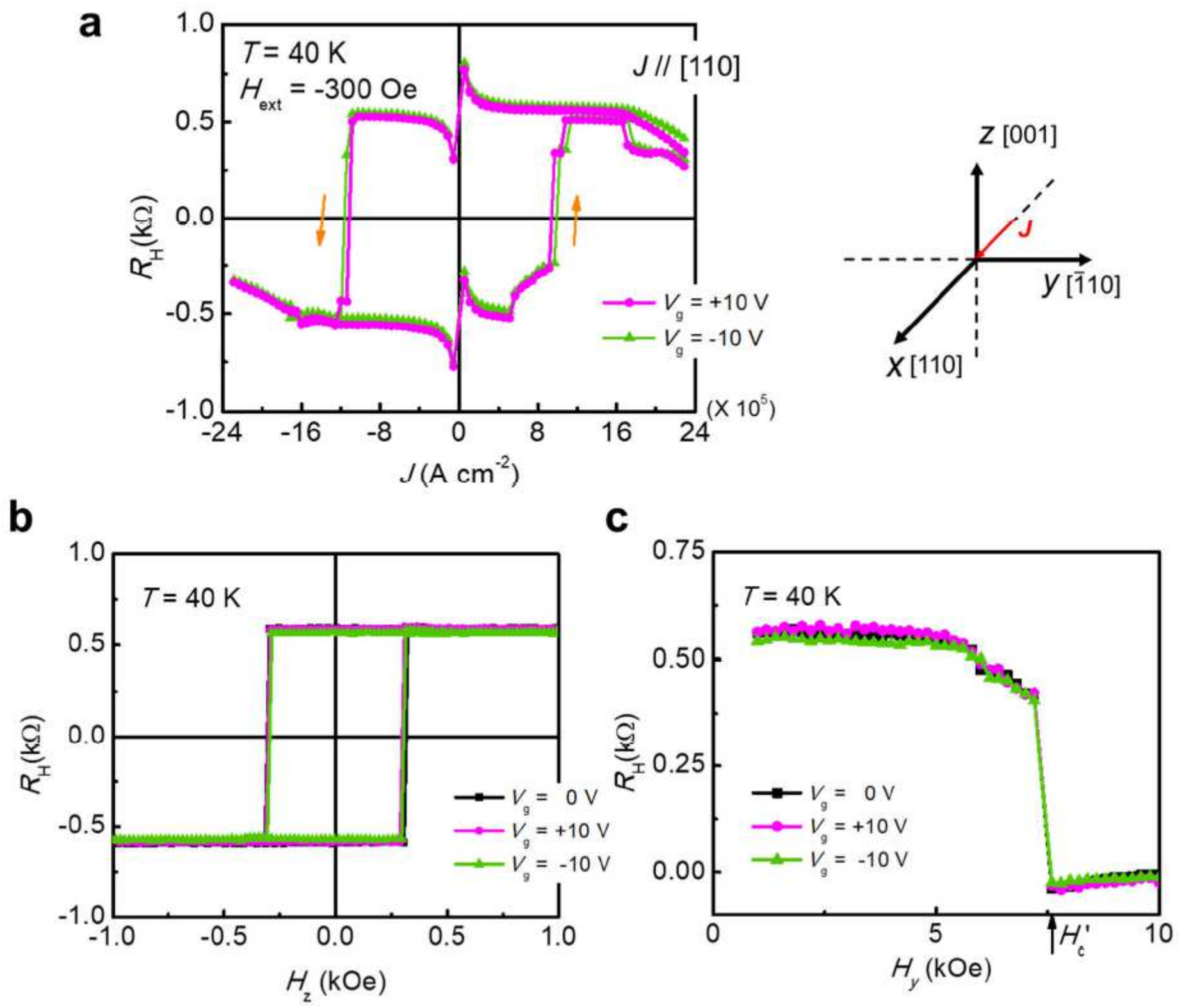

Figure 5

Vg dependence of SOT switching with $\mathrm{J} / /$ ["110" ], Hc, and Han. a, Field-assisted SOT switching with Vg of 0 and $\pm 10 \mathrm{~V}$ in a 7-nm-thick (Ga0.94,Mn0.06)As thin film at $40 \mathrm{~K}$. Here, $\mathrm{J} / /[110]$ and Hext $=-300$ Oe. b, Out-of-plane magnetic field $\mathrm{Hz}$ dependence and c, in-plane magnetic field Hy dependence of the Hall

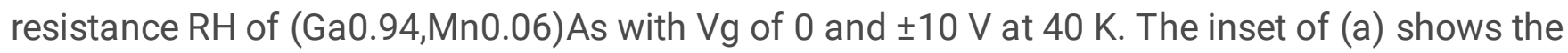
coordinate relationship. (Source data are provided as a Source Data file.)

\section{Supplementary Files}

This is a list of supplementary files associated with this preprint. Click to download.

- supplementaryElectricfieldcontrolofSOTswitching1112.docx 